\title{
Scaling Laws for Line Networks: From Zero-Error to Min-Cut Capacity
}

\author{
Urs Niesen \\ MIT \\ Email: uniesen@mit.edu
}

\author{
Christina Fragouli \\ EPFL \\ Email: christina.fragouli@epfl.ch
}

\author{
Daniela Tuninetti \\ UIC \\ Email: daniela@ece.uic.edu
}

\begin{abstract}
We consider communication through a cascade of $L$ identical Discrete Memoryless Channels (DMCs). The source and destination node are allowed to use coding schemes of arbitrary complexity, but the intermediate relay nodes are restricted to process only blocks of $N$ symbols. It is well known that for any $L$ and $N \rightarrow \infty$ the relays can use a capacity achieving code and communicate reliably as long as the rate of this code is below the capacity of the underlying DMC. The capacity of the cascade is hence equal to the network min-cut capacity. For finite $N$ and $L \rightarrow \infty$, we showed in previous work that the optimal intermediate processing is the highest rate zero-error code of length $N$ for the underlying DMC. The capacity of the cascade coincides with the rate of this zero-error code, and is always below the zero-error capacity. In this work, we characterize how $N$ must scale with $L$ in order to achieve rates in between the zero-error and the min-cut capacity.

In particular, we have observed that $N=\Theta(\log L)$ is sufficient to achieve any rate below the min-cut capacity. Here, we develop a novel upper bound on the capacity of cascades with optimal intermediate processing that applies for any $(N, L)$ pairs and use it to show that $N=\Theta(\log L)$ is necessary to achieve certain rates above the zero-error capacity. Furthermore, we propose a method to evaluate our upper bound by establishing a connection with the Set-Cover Problem in algorithms.
\end{abstract}

\section{INTRODUCTION}

Communication systems today are organized in large scale networks, with Internet the most conspicuous example, where information needs to traverse multiple hops in order to reach its destination. Another such example are wireless ad-hoc networks where the average number of hops between a sourcedestination pair scales as the square root of the number of nodes in the network [4]. Each of the hops may introduce errors, which become more and more pronounced as the size of the network grows.

Motivated by these observations, in [2] we investigated what benefits finite complexity processing at intermediate nodes may offer. We modeled the communication path between the source and the destination as a line network that consists of $L$ cascaded identical DMCs, and allowed each intermediate node to process blocks of $N$ symbols. This is a reasonable definition of complexity as it allows to bound not only processing complexity, but also delay, and memory requirements at intermediate nodes. Moreover, it is well suited to an environment where information is transmitted in packets.

In [2] we showed that if the network length increases $(L \rightarrow \infty)$ but the blocklength $N$ is fixed, the optimal processing is identical at each relay and corresponds to an optimal (highest rate) zero-error code of length $N$. Thus, the capacity of the cascade coincides with the rate of this code and it is hence always below the zero-error capacity of the underlying channel. The zero-error capacity is the maximum rate at which information can be communicated over a channel with zero probability of error [5]. An intuitive interpretation of this result is that, as $L \rightarrow \infty$, the zero-error capacity is the only part of the transmitted information rate that we may hope to preserve. On the other hand, when $N \rightarrow \infty$ the relays can use a capacity achieving code and communicate reliably as long as the rate of this code is below the capacity of the DMC. That is, for $N \rightarrow \infty$ we can achieve the min-cut capacity [1].

Since the zero-error capacity and the min-cut capacity might differ quite substantially, a natural question to ask is what happens if we allow $N$ to grow with $L$. In this paper we investigate how $N$ needs to scale with $L$ in order to achieve rates above the zero-error capacity. In [3] we showed that $N=$ $\Theta(\log L)^{1}$ is sufficient to achieve any rate below the min-cut capacity. Here, we show that $N=\Theta(\log L)$ is necessary to achieve certain rates above the zero-error capacity.

In order to prove our result, we start by deriving a novel upper bound on the capacity of cascades with optimal intermediate processing that is valid for any $L$ and $N$. We decompose the channel transition matrix into a linear combination of two stochastic matrices. We then develop a bound that depends on this decomposition through the smallest rank of these two matrices. With this bound we show that logarithmic scaling of $N$ with $L$ is necessary to achieve certain rates above the zero-error capacity.

We also show that finding the minimum rank decomposition of a channel transition matrix is equivalent to solving a Set Cover Problem, for which polynomial time approximation algorithms are available in the literature. Using such algorithms allows us to compute a decomposition of the channel transition matrix whose gap from the optimal minimum rank decomposition vanishes as $N$ increases (recall that here we are interested in non-finite $N$ ). Interestingly, the Set-Cover Problem is the "dual" (in a sense to be made precise later) of the Maximum Independent Set Problem, whose solution leads to the channel zero-error capacity.

The paper is organized as follows. Section II introduces

\footnotetext{
${ }^{1}$ We use Knuth's notation: $f(n)=O(g(n))$ means that there exists a constant $c$ and integer $n_{0}$ such that $f(n) \leq c g(n)$ for $n>n_{0}$ $f(n)=\Theta(g(n))$ denotes that $f(n)=O(g(n))$ as well as $g(n)=O(f(n))$.
} 
the network model and briefly reviews our previous results. Section III presents the upper bound on the capacity that is used in Section IV to derive the scaling law. Section V concludes the paper.

\section{Model And Previous Results}

\section{A. Model}

We consider line networks with $L-1$ relays as depicted in Figure 1. The source $A_{0}$ sends information to the destination $A_{L}$ via relays $\left\{A_{i}\right\}_{i=1}^{L-1}$. Each link corresponds to the same DMC with finite input alphabet $\mathcal{X}$, finite output alphabet $\mathcal{Y}$, and arbitrary transition probability matrix $\boldsymbol{V}$. We assume that all the DMCs in the cascade are the same.

We restrict the relays $\left\{A_{i}\right\}_{i=1}^{L-1}$ to perform operations from blocks of $N$ symbols in $\mathcal{Y}$ to blocks of $N$ symbols in $\mathcal{X}$ in a memoryless fashion across blocks. Using $N$ times the channel $\boldsymbol{V}$ between $A_{i}$ and $A_{i+1}$, amounts to connecting $A_{i}$ and $A_{i+1}$ through an equivalent DMC with input alphabet $\mathcal{X}^{N}$, output alphabet $\mathcal{Y}^{N}$, and transition probability matrix $\boldsymbol{V}^{\otimes N}$ where $\otimes$ denotes the Kronecker product. For the node $A_{i}$, we denote by $\boldsymbol{X}_{i} \in \mathcal{X}^{N}$ what the relay sends and with $\boldsymbol{Y}_{i} \in \mathcal{Y}^{N}$ what the relay receives. The output $\boldsymbol{X}_{i}$ is then a (not necessarily deterministic) function of $\boldsymbol{Y}_{i}$. This function can be described by a transition probability matrix $\boldsymbol{M}_{i}$ specifying for each realization $\boldsymbol{x}$ of $\boldsymbol{X}_{i}$ and $\boldsymbol{y}$ of $\boldsymbol{Y}_{i}$ the probability $\operatorname{Pr}\left[\boldsymbol{X}_{i}=\boldsymbol{x} \mid \boldsymbol{Y}_{i}=\boldsymbol{y}\right]$.

We allow the source $A_{0}$ and the destination $A_{L}$ to perform coding and decoding of arbitrary complexity, across a possibly infinite number of symbols in $\mathcal{X}^{N}$ and $\mathcal{Y}^{N}$.

We are interested in identifying the set of processings $\left\{\boldsymbol{M}_{i}\right\}_{i=1}^{L-1}$ that achieve

$$
C_{N, L}(\boldsymbol{V}) \triangleq \max _{\left\{\boldsymbol{M}_{i}\right\}_{i=1}^{L-1}} \frac{1}{N} C\left(\boldsymbol{V}^{\otimes N} \prod_{i=1}^{L-1}\left(\boldsymbol{M}_{i} \boldsymbol{V}^{\otimes N}\right)\right) .
$$

Here, $C(\boldsymbol{Q})=\max _{\boldsymbol{p}} I(\boldsymbol{p}, \boldsymbol{Q})$ where $\boldsymbol{p}$ is the input distribution and $Q$ the channel transition matrix.

In this paper, we will also use the notion of the zero-error capacity of the underlying channel $\boldsymbol{V}$. The zero-error capacity is defined in [5] as the maximum rate at which communication is possible with zero probability of error and can be computed as follows. For a channel with transition matrix $\boldsymbol{V}$, we call two input letters $k$ and $\ell$ adjacent if there exists an output letter $j$ such that $[\boldsymbol{V}]_{k, j}>0$ and $[\boldsymbol{V}]_{\ell, j}>0$. We then construct a graph $G(\boldsymbol{V})$ corresponding to the stochastic matrix $\boldsymbol{V}$ having as vertex set the possible inputs of $\boldsymbol{V}$ and in which two vertices are connected by an edge if the corresponding input letters are adjacent. Denote by $M_{0}(\boldsymbol{V})$ the largest number of input letters of $\boldsymbol{V}$ no two of which are adjacent. This integer is known in graph theory as the independence number of graph $G(\boldsymbol{V})$. The zero-error capacity of $\boldsymbol{V}$ is

$$
C_{0}(\boldsymbol{V}) \triangleq \sup _{n} \frac{1}{n} \log M_{0}\left(\boldsymbol{V}^{\otimes n}\right) .
$$

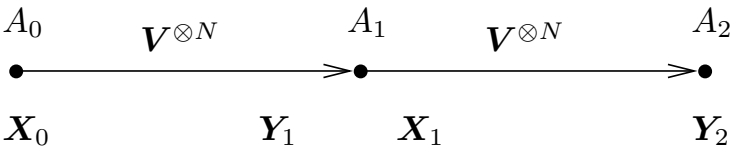

Fig. 1. A line network with two channels and one relay $(L=2)$.

\section{B. Previous Results}

Clearly, for any DMC with transition probability matrix $\boldsymbol{V}$, any intermediate processing of length $N$ and any network length $L$, we have

$$
\frac{1}{N} \log M_{0}\left(\boldsymbol{V}^{\otimes N}\right) \leq C_{N, L}(\boldsymbol{V}) \leq C(\boldsymbol{V}) .
$$

The lower bound in (1) is achievable by using the same zeroerror code of length $N$ at each node in the network, while the upper bound in (1) is achievable by using a capacity achieving code at each node in the network. That no other coding strategy can do better than $C(\boldsymbol{V})$ is clear from the min-cut bound. Hence, the upper bound in (1) is tight for $N \rightarrow \infty$ [1].

Our main result in [2] states that the lower bound in (1) is tight for finite $N$ and $L \rightarrow \infty$ :

\section{Theorem II.1 (Allerton 2005).}

$$
\lim _{L \rightarrow \infty} C_{N, L}(\boldsymbol{V})=\frac{1}{N} \log M_{0}\left(\boldsymbol{V}^{\otimes N}\right) \leq C_{0}(\boldsymbol{V})
$$

This theorem tells us that the $\operatorname{limit}_{\lim _{L \rightarrow \infty} C_{N, L}(\boldsymbol{V}) \text { exists }}$ and that the optimal processing is identical at each node in the limit of large $L$. This processing corresponds to the best, in the sense of highest rate, zero-error code of length $N$ for the channel $\boldsymbol{V}$. Thus, the capacity of the infinite cascade equals the rate of this zero-error code and is always upper bounded by the zero-error capacity of $\boldsymbol{V}$. Notice that any rate strictly below the zero-error capacity is achievable with finite length processing.

Since $C_{0}(\boldsymbol{V})$ and $C(\boldsymbol{V})$ might differ quite substantially, a natural question to ask is what happens if both $N$ and $L$ are allowed to grow. In [3] we derived the following lower bound on $C_{N, L}(\boldsymbol{V})$ :

\section{Theorem II.2 (ISITA 2004).}

$$
C_{N, L}(\boldsymbol{V}) \geq \max _{r \in[0, C(\boldsymbol{V})]} r(1-\exp (-N E(r)))^{L}-\frac{1}{N},
$$

where $E(r)$ is the random coding error exponent for the channel $\boldsymbol{V}$ as a function of the rate $r$.

The bound in Theorem II.2 is tight for $N \rightarrow \infty$ [6].

\section{UPPER BOUND}

In this section, we derive an upper bound on $C_{N, L}(\boldsymbol{V})$ that applies for all values of $N$ and $L$. We then use this upper bound and the lower bound in Theorem II.2 to show that a processing length of $N=\Theta(\log L)$ is sufficient to achieve

$$
C_{N, L}(\boldsymbol{V}) \geq R(\alpha) \triangleq(1-\alpha) C_{0}(\boldsymbol{V})+\alpha C(\boldsymbol{V})
$$

for all $\alpha \in[0,1]$ and necessary for all $\alpha \in[\beta, 1]$ with $\beta \leq 1$. 
Our upper bound for $C_{N, L}(\boldsymbol{V})$ is expressed as a linear combination of the min-cut capacity and of a term reminiscent of the limiting capacity $\frac{1}{N} \log M_{0}\left(\boldsymbol{V}^{\otimes N}\right)$ derived in Theorem II.1. The basic idea is to decompose the equivalent channel transition matrix $\boldsymbol{V}^{\otimes N}$ into a linear combination of two stochastic matrices, one of which has rank as close as possible to $M_{0}\left(\boldsymbol{V}^{\otimes N}\right)$. We also discuss efficient algorithms to determine such a decomposition.

Theorem III.1. For any stochastic matrix $\boldsymbol{V}$ and any integer $N$, if there exist two stochastic matrices $\boldsymbol{A}_{N}$ and $\boldsymbol{B}_{N}$, and $\delta_{N} \in(0,1]$ such that

$$
\boldsymbol{V}^{\otimes N}=\delta_{N} \boldsymbol{A}_{N}+\left(1-\delta_{N}\right) \boldsymbol{B}_{N}
$$

then

$$
\begin{aligned}
C_{N, L}(\boldsymbol{V}) \leq & \left(1-\left(1-\delta_{N}\right)^{L-1}\right) \frac{\log \operatorname{rank}\left(\boldsymbol{A}_{N}\right)}{N} \\
& +\left(1-\delta_{N}\right)^{L-1} C(\boldsymbol{V}) .
\end{aligned}
$$

Proof. Assume (3) holds, then,

$$
\begin{aligned}
C_{N, L}(\boldsymbol{V}) & =\frac{1}{N} C\left(\boldsymbol{V}^{\otimes N} \prod_{i=1}^{L-1}\left(\boldsymbol{M}_{i} \boldsymbol{V}^{\otimes N}\right)\right) \\
& =\frac{1}{N} C\left(\left(\delta_{N} \boldsymbol{A}_{N}+\left(1-\delta_{N}\right) \boldsymbol{B}_{N}\right) \prod_{i=1}^{L-1}\left(\boldsymbol{M}_{i} \boldsymbol{V}^{\otimes N}\right)\right) \\
& \stackrel{(\text { a) }}{\leq} \delta_{N} \frac{1}{N} C\left(\boldsymbol{A}_{N} \prod_{i=1}^{L-1}\left(\boldsymbol{M}_{i} \boldsymbol{V}^{\otimes N}\right)\right) \\
& +\left(1-\delta_{N}\right) \frac{1}{N} C\left(\left(\boldsymbol{B}_{N} \boldsymbol{M}_{1}\right) \boldsymbol{V}^{\otimes N} \prod_{i=2}^{L-1}\left(\boldsymbol{M}_{i} \boldsymbol{V}^{\otimes N}\right)\right) \\
& \stackrel{\text { (b) }}{\leq} \delta_{N} \frac{C\left(\boldsymbol{A}_{N}\right)}{N}+\left(1-\delta_{N}\right) \frac{1}{N} C_{N, L-1}(\boldsymbol{V})
\end{aligned}
$$

where the inequality (a) follows from the convexity of mutual information in the channel transition matrix and (b) follows from the data processing inequality. By repeating the same argument we get

$C_{N, L}(\boldsymbol{V}) \leq\left(1-\left(1-\delta_{N}\right)^{L-1}\right) \frac{C\left(\boldsymbol{A}_{N}\right)}{N}+\left(1-\delta_{N}\right)^{L-1} C(\boldsymbol{V})$.

We can further upper bound $C\left(\boldsymbol{A}_{N}\right)$ by the logarithm of the rank of $\boldsymbol{A}_{N}$ [7] to yield (4).

In order to obtain the best bound for any given $N, \boldsymbol{A}_{N}$ should be chosen to have the smallest rank possible. A possible choice is $\boldsymbol{A}_{N}=\boldsymbol{A}_{1}^{\otimes N}$ and $\delta_{N}=\delta_{1}^{N}$ which gives

$$
\begin{aligned}
C_{N, L}(\boldsymbol{V}) \leq & \left(1-\left(1-\delta_{1}^{N}\right)^{L-1}\right) \log \operatorname{rank}\left(\boldsymbol{A}_{1}\right) \\
& +\left(1-\delta_{1}^{N}\right)^{L-1} C(\boldsymbol{V})
\end{aligned}
$$

since $\operatorname{rank}\left(\boldsymbol{A}_{N}\right)=\left(\operatorname{rank}\left(\boldsymbol{A}_{1}\right)\right)^{N}$. However, this choice does not give the best possible bound in general [6].

Notice that, for any matrix $\boldsymbol{A}_{N}$ such that (3) holds, the inequality in (4) and Theorem II.1 imply

$$
\frac{1}{N} \log M_{0}\left(\boldsymbol{V}^{\otimes N}\right) \leq \frac{1}{N} \log \operatorname{rank}\left(\boldsymbol{A}_{N}\right) .
$$

It can be shown that if, for some $N$, we find a decomposition such that $\operatorname{rank}\left(\boldsymbol{A}_{N}\right)=M_{0}\left(\boldsymbol{V}^{\otimes N}\right)$, then $C_{0}(\boldsymbol{V})=$ $\frac{1}{N} \log \operatorname{rank}\left(\boldsymbol{A}_{N}\right)$. In this case, the bound in (4) is tight in the limit as $L \rightarrow \infty$ and the decay of $C_{N, L}(\boldsymbol{V})$ to the limiting capacity is exponentially fast in $L$. In [2] we showed that if we impose that $\boldsymbol{M}_{i}=\boldsymbol{M}$ for all $i \in\{1, \ldots, L-1\}$ (identical processing), then the limiting capacity is equal to the logarithm on the number of eigenvalues of modulus one of $\boldsymbol{V}^{\otimes N} \boldsymbol{M}$ and the limit is approached exponentially fast in $L$. If $\operatorname{rank}\left(\boldsymbol{A}_{N}\right)=M_{0}\left(\boldsymbol{V}^{\otimes N}\right)$, then the exponential decay also applies to non identical processing. This implies that even for long, but not infinite, cascades the derived limiting result in Theorem II.1 is meaningful. It also highlights the importance of well chosen intermediate processing. The exponent that can be derived from (4) in the case $\operatorname{rank}\left(\boldsymbol{A}_{N}\right)=M_{0}\left(\boldsymbol{V}^{\otimes N}\right)$, namely

$\lim _{L \rightarrow \infty}-\frac{1}{L} \log \left(C_{N, L}(\boldsymbol{V})-\frac{1}{N} \log M_{0}\left(\boldsymbol{V}^{\otimes N}\right)\right) \geq-\log \left(1-\delta_{N}\right)$,

is however not tight in general [6].

The problem of finding the matrix $\boldsymbol{A}_{N}$ with minimum rank is equivalent to the Set Cover Problem described as follows [8]. Given a universe $\mathcal{U}$ of $n$ elements, a collection $\mathcal{S}=\left\{\mathcal{S}_{1} \ldots \mathcal{S}_{m}\right\}$ of subsets of $\mathcal{U}$, and a cost function for each subset in $\mathcal{S}$, find a minimum cost subcollection of $\mathcal{S}$ that covers all the elements in $\mathcal{U}$. This problem can be formulated as an integer program by assigning a variable $x_{i}$ for each set $\mathcal{S}_{i} \in \mathcal{S}$, where $x_{i}=1$ if set $\mathcal{S}_{i}$ takes part in the subcollection and $x_{i}=0$ otherwise. The constraint is that every element in $\mathcal{U}$ must belong to at least one of the picked sets $\mathcal{S}_{i}$. The set cover problem and its Linear Program (LP) relaxation (Primal) are provided in Table I, for the special case where the cost of all sets is one, which is the case of interest here. A variety of approximation algorithms are available in the literature for the set cover problem [8]. Those algorithms run in polynomial time in $n$ and provide approximations with gap at most $\log n$ from the optimal solution.

In our case, the universe $\mathcal{U}$ is the set of $n=|\mathcal{X}|^{N}$ inputs of the channel $\boldsymbol{V}^{\otimes N}$. The set of $m=|\mathcal{Y}|^{N}$ outputs defines $\mathcal{S}$, in that the subset $\mathcal{S}_{i}$ contains the inputs that result with nonzero probability in output $i$, for $i=1, \ldots, m$. We are going to show that the minimum rank matrix $\boldsymbol{A}_{N}$ we can find has rank $\mathrm{OPT}_{A_{N}}$, if and only if $\mathrm{OPT}_{A_{N}}$ is the minimum cost of the described set cover problem.

Consider a solution of the set cover problem. This provides us with a set of outputs that cover all inputs. Construct a matrix $\boldsymbol{A}_{N}$ as follows. Take the binary matrix $\left\lceil\boldsymbol{V}^{\otimes N}\right\rceil$, where the ceiling operation is component-wise. Replace with the all-zero columns those columns of $\left\lceil\boldsymbol{V}^{\otimes N}\right\rceil$ that correspond to outputs where $x_{i}=0$ in the set cover problem. Then normalize all rows so that they sum to one. The matrix $\boldsymbol{A}_{N}$ is a valid stochastic matrix because its nonzero columns "cover" all inputs, i.e., each row has at least one nonzero element. Moreover, $\boldsymbol{A}_{N}$ contains exactly $\mathrm{OPT}_{A_{N}}$ nonzero columns. These columns are linearly independent, otherwise $\mathrm{OPT}_{A_{N}}$ would not be an optimal solution. Thus $\operatorname{rank}\left(\boldsymbol{A}_{N}\right)=\mathrm{OPT}_{A_{N}}$. 
TABLE I

MAXimum IndePENDENT SET PROBlem, SET COVER PROBlem, AND THEIR LP RELAXATIONS

\begin{tabular}{|c|c|}
\hline $\begin{array}{l}\text { Set Cover Problem } \\
\min \sum_{i} x_{i} \\
\sum_{i: u \in \mathcal{S}_{i}} x_{i} \geq 1, \forall u \in \mathcal{U} \\
x_{i} \in\{0,1\}, \quad \forall i\end{array}$ & $\begin{array}{l}\text { LP Relaxation (Primal) } \\
\min \sum_{i} x_{i} \\
\sum_{i: u \in \mathcal{S}_{i}} x_{i} \geq 1, \forall u \in \mathcal{U} \\
0 \leq x_{i} \leq 1, \quad \forall i\end{array}$ \\
\hline $\begin{array}{l}\text { Max Independent Set Problem } \\
\max \sum_{j} y_{j} \\
\sum_{j \in \mathcal{S}_{i}} y_{j} \leq 1 \forall \mathcal{S}_{i} \in \mathcal{S} \\
y_{j} \in\{0,1\}, \quad \forall j\end{array}$ & $\begin{array}{l}\text { LP Relaxation (Dual) } \\
\max \sum_{j} y_{j} \\
\sum_{j \in \mathcal{S}_{i}} y_{j} \leq 1 \forall \mathcal{S}_{i} \in \mathcal{S} \\
0 \leq y_{j} \leq 1, \quad \forall j\end{array}$ \\
\hline
\end{tabular}

Choose $\delta_{N}$ to be the largest number such that the matrix

$$
\boldsymbol{B}_{N}=\frac{1}{1-\delta_{N}}\left(\boldsymbol{V}^{\otimes N}-\delta_{N} \boldsymbol{A}_{N}\right)
$$

is a valid stochastic matrix. It is easy to see that this $\delta_{N}$ is at least as large as the minimum of the entries of $\boldsymbol{V}^{\otimes N}$ that correspond to a non-zero entry in $\boldsymbol{A}_{N}$.

For the reverse direction, note that we can always take matrix $\boldsymbol{A}_{N}$ to be binary. Moreover, the requirement that $\boldsymbol{A}_{N}$ and $\boldsymbol{B}_{N}$ are stochastic implies that $\boldsymbol{A}_{N}$ has either zero columns, or columns from $\left\lceil\boldsymbol{V}^{\otimes N}\right\rceil$. The result follows.

By using one of the available approximation algorithms, we can calculate in polynomial time in $n$ a matrix $\boldsymbol{A}_{N}$ that has rank bigger than the minimum by a factor of at $\operatorname{most} \log n=$ $N \log |\mathcal{X}|$. As we are interested in $\frac{1}{N} \log \left(\operatorname{rank}\left(\boldsymbol{A}_{N}\right)\right)$ this implies that the loss we incur by using these approximation algorithms to find an $\boldsymbol{A}_{N}$ matrix vanishes as $N \rightarrow \infty$.

The problem of finding the matrix $\boldsymbol{A}_{N}$ with minimum rank is closely related to computing $M_{0}\left(\boldsymbol{V}^{\otimes N}\right)$. To see this, consider the (strong) LP dual of the set cover LP relaxation described in Table I. The dual LP is the LP relaxation of the Maximum Independent Set Problem, which takes as input a graph adjacency matrix and calculates the graph independence number. This is the largest number of vertices in the graph no two of which are connected by an edge. This maximum independent set problem can be formulated as an integer program as follows. Assign a variable $y_{j}$ for each vertex of the graph, $y_{j}=1$ if the vertex takes part in the independent set and $y_{j}=0$ otherwise. The constraint is that no two picked vertices are connected with an edge.

For our purposes we use the adjacency matrix corresponding to the graph $G\left(\boldsymbol{V}^{\otimes N}\right)$. The solution of the maximum independent set problem for $G\left(\boldsymbol{V}^{\otimes N}\right)$ directly gives an optimal zero-error code of length $N$ for the channel $\boldsymbol{V}$. Denote by $\mathrm{OPT}_{M_{0}}$ this optimal solution. Obviously, $\mathrm{OPT}_{A_{N}}$ is lowerbounded by $\mathrm{OPT}_{M_{0}}$. In fact, $\mathrm{OPT}_{A_{N}}$ is the minimum number of outputs such that all inputs are covered. Since all inputs are covered, this implies that any $\mathrm{OPT}_{A_{N}}+1$ inputs have at least one output in common. Thus $\mathrm{OPT}_{M_{0}} \leq \mathrm{OPT}_{A_{N}}$, which provides an alternative derivation of Eq. (5).

Note that, in the instances where the integrality gap between the maximum independent set problem and its LP relaxation is small, then there exists a matrix $\boldsymbol{A}_{N}$ such that $\frac{1}{N} \log \operatorname{rank}\left(\boldsymbol{A}_{N}\right) \approx \frac{1}{N} \log M_{0}\left(\boldsymbol{V}^{\otimes N}\right)$, i.e., the inequality in (5) becomes an equality with all the discussed consequences that this entails.

\section{SCALING LAWS}

In this section, we show that logarithmic growth of $N$ with $L$ is sufficient, and in many cases also necessary, to achieve rates above the zero-error capacity.

Recall that any rate strictly below the zero-error capacity can be achieved with finite blocklength processing. Here we are interested in rates that indeed need an infinite blocklength. For any $\alpha \in[0,1]$ and for $R(\alpha)$ as in (2), we define

$$
N^{\star}(L, \alpha) \triangleq \min \left\{N: C_{N, L}(\boldsymbol{V}) \geq R(\alpha)\right\} .
$$

The next theorem gives an upper bound on $N^{\star}(L, \alpha)$, establishing that logarithmic growth of $N$ with $L$ is sufficient to achieve $R(\alpha)$.

Theorem IV.1. For every $\varepsilon>0$ and for all $N \geq \frac{1}{\varepsilon}$

$$
N^{\star}(L, \alpha) \leq \inf _{r \in(R(\alpha)+\varepsilon, C(\boldsymbol{V})]} \frac{1}{E(r)} \log \left(\frac{L}{1-\frac{R(\alpha)+\varepsilon}{r}}\right)
$$

is sufficient to achieve $R(\alpha)=(1-\alpha) C_{0}(\boldsymbol{V})+\alpha C(\boldsymbol{V})$.

Proof. Theorem II.2 asserts that for any $r \in[0, C(\boldsymbol{V})]$

$$
\begin{aligned}
C_{N, L}(\boldsymbol{V}) & \geq r(1-\exp (-N E(r)))^{L}-\frac{1}{N} \\
& \geq r(1-L \exp (-N E(r)))-\frac{1}{N} .
\end{aligned}
$$

Since the right hand side of (6) is always smaller than $r$, in order to attain $R(\alpha), r$ must satisfy $r>R(\alpha)$. Setting the right hand side of (6) to be greater than or equal to $R(\alpha)$, we get

$$
\log \left(1-\frac{R(\alpha)+\frac{1}{N}}{r}\right) \leq \log (L)-N E(r) .
$$

Thus for all $N \geq \frac{1}{\varepsilon}$

$$
N \leq \frac{1}{E(r)}\left(\log (L)-\log \left(1-\frac{R(\alpha)+\varepsilon}{r}\right)\right) .
$$

But since this is true for all $r \in(R(\alpha)+\varepsilon, C(\boldsymbol{V})]$, we can minimize the right hand side of (7) over $r$ to get the tightest bound.

The next theorem establishes that logarithmic growth of $N$ with $L$ is necessary to achieve $R(\alpha)$ for all $\alpha \geq \beta_{m}$ where $\beta_{m}$ is a non-negative constant.

\section{Theorem IV.2.}

$$
N^{\star}(L, \alpha) \geq \frac{\log (L-1)-\log \log \frac{1}{\alpha-\beta_{m}}}{\frac{1}{m} \log \frac{1}{\delta_{m}}}
$$

for all

$$
\alpha \geq \beta_{m} \triangleq \frac{\frac{1}{m} \log \operatorname{rank}\left(\boldsymbol{A}_{m}\right)-C_{0}(\boldsymbol{V})}{C(\boldsymbol{V})-C_{0}(\boldsymbol{V})},
$$

where $m$ is any integer such that the stochastic matrices $\boldsymbol{A}_{m}, \boldsymbol{B}_{m}$, and the real-valued constant $\delta_{m} \in(0,1]$ in the decomposition $\boldsymbol{V}^{\otimes m}=\delta_{m} \boldsymbol{A}_{m}+\left(1-\delta_{m}\right) \boldsymbol{B}_{m}$ satisfy $\frac{1}{m} \log \operatorname{rank}\left(\boldsymbol{A}_{m}\right) \leq C(\boldsymbol{V})$. 
Proof. From Theorem III.1,

$$
\begin{aligned}
C_{N, L}(\boldsymbol{V}) \leq & \left(1-\left(1-\delta_{m}^{N / m}\right)^{L-1}\right) \frac{1}{m} \log \operatorname{rank}\left(\boldsymbol{A}_{m}\right) \\
& +\left(1-\delta_{m}^{N / m}\right)^{L-1} C(\boldsymbol{V}) .
\end{aligned}
$$

In order to achieve $R(\alpha)$ it is hence necessary that

$$
\begin{aligned}
(1-\alpha) C_{0}(\boldsymbol{V}) & +\alpha C(\boldsymbol{V}) \leq\left(1-\delta_{m}^{N / m}\right)^{L-1} C(\boldsymbol{V}) \\
& +\left(1-\left(1-\delta_{m}^{N / m}\right)^{L-1}\right) \frac{1}{m} \log \operatorname{rank}\left(\boldsymbol{A}_{m}\right) .
\end{aligned}
$$

By re-arranging the different terms, we get

$$
\alpha-\beta_{m} \leq\left(1-\delta_{m}^{N / m}\right)^{L-1} \underbrace{\frac{C(\boldsymbol{V})-\frac{1}{m} \log \operatorname{rank}\left(\boldsymbol{A}_{m}\right)}{C(\boldsymbol{V})-C_{0}(\boldsymbol{V})}}_{\triangleq \gamma_{m}} .
$$

If the value of $m$ is such that the hypothesis of the theorem are satisfied we have $\beta_{m} \in[0,1]$ and $\gamma_{m} \in[0,1]$, and hence

$$
\left(1-\delta_{m}^{N / m}\right)^{L-1} \geq \alpha-\beta_{m} .
$$

By using the fact that $-\log (1-x) \geq x$ for all $x \in[0,1]$, we get that for all $\alpha \geq \beta_{m}$

$$
\log \frac{1}{\alpha-\beta_{m}} \geq(L-1) \log \frac{1}{1-\delta_{m}^{N / m}} \geq(L-1) \delta_{m}^{N / m},
$$

and hence

$$
N \frac{1}{m} \log \frac{1}{\delta_{m}} \geq \log (L-1)-\log \log \frac{1}{\alpha-\beta_{m}},
$$

from which the result in (8) follows.

Notice that, since we are interested in the regime $N \gg 1$, the assumptions of the theorem about the integer $m$ can be relaxed to $\lim _{m \rightarrow \infty} \frac{1}{m} \log \operatorname{rank}\left(\boldsymbol{A}_{m}\right) \leq C(\boldsymbol{V})$.

The following examples illustrate the use of Theorems IV.1 and IV.2.

\section{Example IV.1. [Binary Symmetric Channel]}

Consider a cascade of $L$ binary symmetric channels with crossover probability $p$. For this channel $C_{0}(\boldsymbol{V})=0$ and hence we get from Theorem II.1 that any finite length processing performed at every node in the network will result in a zero end-to-end rate as $L \rightarrow \infty$. From Theorem IV.1, we see that logarithmic growth of $N$ with $L$ is sufficient to achieve any fraction of the min-cut capacity. We will now show that $N=$ $\Theta(\log L)$ is also necessary to achieve any positive fraction of the min-cut capacity. The equivalent channel matrix $\boldsymbol{V}^{\otimes N}$ has smallest entry $p^{N}$. By collecting $p^{N}$ from all the entries of $\boldsymbol{V}^{\otimes N}$, we can write

$$
\boldsymbol{V}^{\otimes N}=p^{N} \mathbf{1 1}^{T}+\left(1-(2 p)^{N}\right) \boldsymbol{B},
$$

for some stochastic matrix $\boldsymbol{B}$, and where $\mathbf{1}$ is the all one column vector of length $2^{N}$. By using this decomposition we get from Theorem IV.2 that

$$
N^{*}(L, \alpha) \geq \frac{\log (L-1)-\log \log \frac{1}{\alpha}}{\log \frac{1}{\delta}},
$$

with $\delta \triangleq 2 p$. Hence logarithmic growth of $N$ with $L$ is necessary for all positive rates.

\section{Example IV.2. [Pentagon Channel]}

Consider the "pentagon" channel whose transition matrix $\boldsymbol{V}$, for $p \in(0,1)$, is

$$
\boldsymbol{V}=\left(\begin{array}{ccccc}
1-p & p & 0 & 0 & 0 \\
0 & 1-p & p & 0 & 0 \\
0 & 0 & 1-p & p & 0 \\
0 & 0 & 0 & 1-p & p \\
p & 0 & 0 & 0 & 1-p
\end{array}\right)
$$

Here $C(\boldsymbol{V})=\log (5)-H(p)$ and $C_{0}(\boldsymbol{V})=\frac{1}{2} \log (5)$.

For $m=1$ we can find a matrix $\boldsymbol{A}_{1}$ with $\operatorname{rank}\left(\boldsymbol{A}_{1}\right)=$ 3. However, for $m=2$ we can find a matrix $\boldsymbol{A}_{2}$ with $\operatorname{rank}\left(\boldsymbol{A}_{2}\right)=8<\operatorname{rank}\left(\boldsymbol{A}_{1}\right)^{2}=9$. Using the decomposition for $m=2$, we obtain from Theorem IV.2

$$
\beta_{2}=\frac{\frac{1}{2} \log \operatorname{rank}\left(\boldsymbol{A}_{2}\right)-C_{0}(\boldsymbol{V})}{C(\boldsymbol{V})-C_{0}(\boldsymbol{V})}=\frac{\log (8 / 5)}{\log 5-2 H(p)},
$$

showing that logarithmic growth of $N$ with $L$ is necessary to achieve any $R(\alpha)$ with $\alpha \geq \beta_{2}$. With $m=2$, Theorem IV.2 does in this case not allow to state that logarithmic growth is necessary for all $\alpha \in[0,1]$.

\section{COnClusions}

In this paper, we investigated communication through a cascade of $L$ channels, where intermediate nodes can perform processing on blocks of $N$ symbols, and studied how $N$ must scale with $L$ in order to achieve rates above the zero-error capacity. We derived bounds on the capacity of finite length cascades and used them to show that logarithmic growth of $N$ with $L$ is sufficient to achieve any rate below the mincut capacity and necessary to achieve certain rates above the zero-error capacity. We conjecture that logarithmic growth is actually necessary to achieve any rate above the zero error capacity. Proving this conjecture and extending the work to more general networks, traffic configurations and resource constraints is part of ongoing work.

\section{ACKNOWLEDGMENTS}

The authors would like to thank C. Chekuri and D. Shah for helpful discussions.

\section{REFERENCES}

[1] T. Cover, J. Thomas, "Elements of Information Theory," Wiley Series, 1991.

[2] U. Niesen, C. Fragouli, D. Tuninetti, "On the Capacity of an Infinite Cascade of Channels", Allerton 2005, Monticello, IL (USA) Sept. 2005.

[3] D. Tuninetti, C. Fragouli, "Processing along the way: forwarding vs. coding", ISITA 2004, Parma (Italy) Oct. 2004.

[4] P. Gupta, P. R. Kumar, "The Capacity of Wireless Networks", IEEE Trans. on Info. Theory, Vol. 46(2), March 2000.

[5] C. E. Shannon, "The Zero Error Capacity of a Noisy Channel", IEEE Trans. on Info. Theory, Vol. 2(3), Sept. 1956.

[6] U. Niesen, C. Fragouli, D. Tuninetti, "On Capacity of Line Networks", submitted to IEEE Trans. on Info. Theory on Dec. 2005.

[7] C. E. Shannon, "Some Geometrical Results in Channel Capacity", Nachrichtentechnische Zeitschrift, Vol.10, 1957.

[8] V. Vazirani, "Approximation Algorithms", Springer, 2001. 